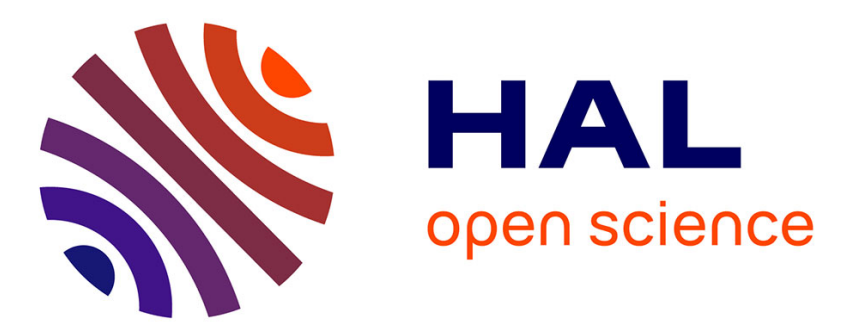

\title{
Vibrational density of states of free and embedded semiconducting GaN nanoparticles
}

Paul Desmarchelier, Konstantinos Termentzidis, Anne Tanguy

\section{To cite this version:}

Paul Desmarchelier, Konstantinos Termentzidis, Anne Tanguy. Vibrational density of states of free and embedded semiconducting GaN nanoparticles. Semiconductor Science and Technology, 2020, 35

(9), pp.094001. 10.1088/1361-6641/ab957c . hal-02992263

\section{HAL Id: hal-02992263 \\ https://hal.science/hal-02992263}

Submitted on 4 May 2021

HAL is a multi-disciplinary open access archive for the deposit and dissemination of scientific research documents, whether they are published or not. The documents may come from teaching and research institutions in France or abroad, or from public or private research centers.
L'archive ouverte pluridisciplinaire HAL, est destinée au dépôt et à la diffusion de documents scientifiques de niveau recherche, publiés ou non, émanant des établissements d'enseignement et de recherche français ou étrangers, des laboratoires publics ou privés. 


\title{
Vibrational density of states of free and embedded semiconducting GaN nanoparticles
}

\author{
P Desmarchelier ${ }^{12}$, K Termentzidis ${ }^{1}$, A Tanguy ${ }^{2}$ \\ ${ }^{1}$ Universite de Lyon, CETHIL, INSA-Lyon, CNRS UMR5008, F-69621, \\ Villeurbanne, France \\ ${ }^{2}$ Universite de Lyon, LaMCoS, INSA-Lyon, CNRS UMR5259, F-69621, \\ Villeurbanne, France \\ E-mail: konstantinos.termentzidis@insa-lyon.fr
}

\begin{abstract}
The impact of the size of free and embedded GaN nanoparticles on vibrational properties has been studied using 3 different numerical methods. The thermal conductivity of free nanoparticles was also estimated with Equilibrium Molecular Dynamics. Important discrepancies between the vibrational density of states of small nanoparticles compared to the bulk are observed, such as the presence of modes in the bandgap related to the surface modes, the optical peaks decrease, and the redshift of transverse acoustic peak. When these nanoparticles are embedded in a $\mathrm{SiO}_{2}$ matrix, the peaks in the bandgap disappear and the transverse acoustic modes are shifted back to the bulk frequencies. These differences between the free and the embedded nanoparticles tend to disappear for nanoparticles with diameters larger than $5 \mathrm{~nm}$. Finally, the thermal conductivity for free nanoparticles is computed, showing a nonlinear augmentation upon the increase of the size of nanoparticles. The latter results could be useful in effective medium models used to estimate the thermal conductivity of nanocomposites.
\end{abstract}

Submitted to: Semicond. Sci. Technol. 
Vibrational density of states of free and embedded semiconducting GaN nanoparticles 2

\section{Introduction}

Nanoparticles (NPs) consist of a group of nanomaterials with characteristic dimensions of few nanometers and usually a narrow range of size distribution. Thanks to the evolution of nanofabrication methods it is possible to control their sizes, chemical composition and orientation as well as their dispersion characteristics. They can be integrated into a liquid solution [1] or embedded in a solid matrix [2]. There are several studies in the last two decades which show an increasing interest in the topic, notably the influence of embedding NPs in a matrix. For instance, in nanofluids, the viscosity, the electrical conductivity and the density have been found to change with NPs concentration [3]. Metallic NPs have been used to increase the electrical conductivity of polymers [4]. NPs have been proposed also for specific applications, for example nanoplasmonics where metallic NPs are used for coupling electromagnetic and mechanical waves $[5,6]$.

The impact of NPs on the thermal transport starts to attract the scientific interest; from the computation of the thermal properties of the NPs themselves, to their impact on the thermal properties in nanocomposites. Studies showed that the thermal conductivity of single silicon NP represents only a fraction of the bulk conductivity and increases linearly with the diameter $[7,8]$. When the NPs are embedded in a matrix, the effective thermal properties are altered considerably. The role of NPs as scatterers for the heat carriers has been observed [9], but their influence is not trivial: their orientation [10], stiffness [11], or size distribution [12] can have a strong impact on the thermal properties of these nanocomposites.

Concerning the vibrational properties of NPs, they have been first computed using the continuum mechanics solution for the vibrations of a free sphere [13]. This approach was successfully applied to predict resonant modes in NPs down to $1 \mathrm{~nm}$ in diameter for crystalline NPs [14]. This approximation should theoretically work for amorphous NPs as long as characteristic lengthscales of the vibrational modes are not smaller than 40 interatomic distances [15]. Transpositions of the original solution exist for anisotropic materials [16] or embedded NPs [17, 18]. These models based on Lamb's model [19], predict resonant frequencies depending on the material, that scale with the diameter. NPs vibrational properties have been also studied using atomistic models, like molecular dynamics (MD) simulations, with a successful comparison with the continuum theory in the case of $\mathrm{Ge}$ or $\mathrm{Ag}[17,18]$. Some studies have focused on other features of NPs vibrational density of states (VDOS), like spectral spreading and surface effects. These effects were observed in the case of monoatomic metal and bimetallic alloys [20, 21]. The case of III-V semiconductors has been studied by Han and Bester [22, 23], showing similar size effects on NPs VDOS. Finally, II-VI semiconductors NPs have been also studied with a focus on the temperature, size and composition impact on the heat capacity and structural properties [24, 25].

Here MD simulations is used to study the impact of the size of GaN NPs, and whether they are free or embedded in an amorphous $\mathrm{SiO}_{2}$ matrix, on their VDOS. In 
Vibrational density of states of free and embedded semiconducting GaN nanoparticles 3

the following section, the description of the modeling of our configurations and the methodologies to compute the VDOS and the thermal conductivity will be detailed. Section 3 gathers the results of VDOS and participation ratio of free and embedded NPs as well as the thermal conductivity of free NPs. The article ends with the Discussion and Conclusion section in which a comparison of the three VDOS methodologies is analysed.

\section{Materials and methods}

\subsection{Modeling of the configurations}

To create the GaN NPs, we cut them out from a relaxed GaN wurztite crystal. Hereafter, GaN NPs will be referred simply as NPs. Studied NPs radii R ranges between 1 and $4 \mathrm{~nm}$, and contain 369 to 23000 atoms, and they will be labeled as $\mathrm{NP}_{R}$. The free $\mathrm{NPs}_{\mathrm{S}}$ (FNPs) are relaxed in an equilibrium position using a conjugated gradient method, then equilibrated at $300 \mathrm{~K}$ for 20 ps. We use fixed boundary conditions for the simulation box to avoid any artefact due to periodic boundary conditions, such as interactions between neighboring NPs. To model the embedded NPs (ENPs) in a silica matrix a cubic simulation box with length twice this of the NP diameter was cut out in a bulk a-SiO ${ }_{2}$ sample. The box length was chosen here to limit the interactions between neighboring NPs through periodic boundary conditions while limiting the total number of atoms. In this cubic box a spherical pore with a radius larger than the radius of the NPs by $0.1 \AA$ is cut to avoids any superposition of atoms of the matrix with atoms of the NP. Inside this empty pore, we then added the NP, which has been carved out from the bulk GaN crystal. To relax the interfacial stresses, an additional relaxation is performed using the conjugated gradient method. After this step, the system is annealed at $300 \mathrm{~K}$ for $20 \mathrm{ps}$. All modeling and MD simulations are performed using the opensource software LAMMPS [26]. Two configurations representing the two types of materials studied are depicted in figure 1. The Tersoff interaction potential developed by Kioseoglou et al. [27] is used to describe the interatomic interactions. It combines interatomic potential developed: for $\mathrm{SiO}_{2}$ [28], for $\mathrm{GaN}$ [29], for oxynitrides [30] and for silicon nitride [31]. This combined interatomic potential allows the simulations of silica and gallium nitride nanocomposites using the same potential form and it has been used previously with success [10].

The silica glass sample is obtained through the method described by Mantisi et al. [33]. A sample of cristobalite crystal is first created and then relaxed to minimize the potential energy. It is then heated from $0 \mathrm{~K}$ to $5200 \mathrm{~K}$ in $1 \mathrm{~ns}$ in order to melt it, and then is kept during 10 ps for homogenization at this high temperature. It is finally quenched from $5200 \mathrm{~K}$ to $300 \mathrm{~K}$ in 1 ns. All these steps are performed using a BKS interatomic potential [34] instead of the Tersoff potential. The use of a Tersoff potential at high temperature leads to silica micro structure with $40 \%$ coordination defects (five fold or six fold coordinated Si atoms), whereas at the normal pressure range, almost none of them are expected [35]. We then reverted to the Tersoff potential for the quench from 


\section{Vibrational density of states of free and embedded semiconducting GaN nanoparticles 4}
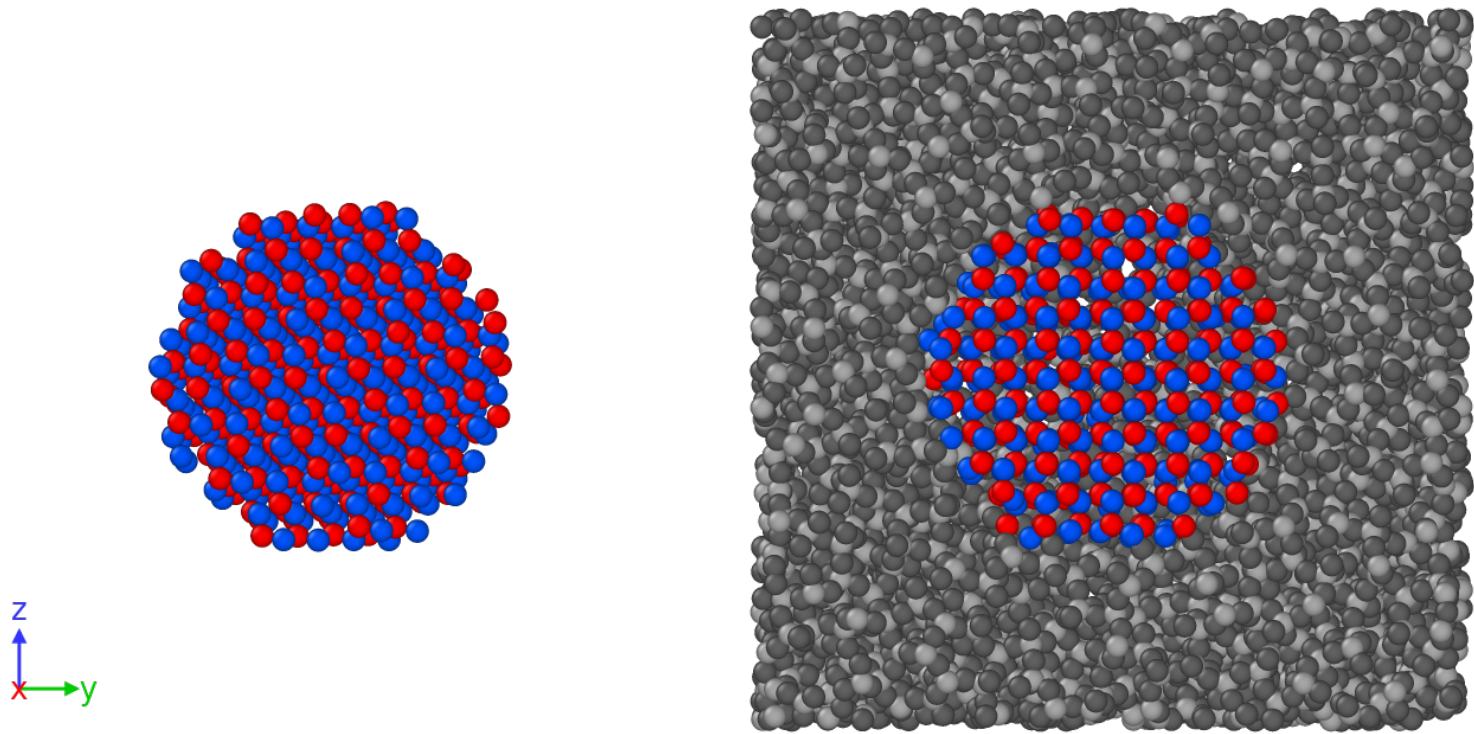

(a)

(b)

Figure 1: Visualisation of the free (a) and embedded NPs in silica matrix (b). Blue atoms are Ga, red N, gray Si and dark gray O, obtained with OVITO [32]

$300 \mathrm{~K}$ to $10 \mathrm{~K}$. Finally we relaxed the structure using a conjugated gradient method and then a Hessian-free truncated Newton algorithm to ensure a better convergence. This method allows to keep the coordination defect below $2 \%$. The partial radial distribution function (PRDF) of the free NPs was examined. The peaks positions and sharpness do not change relative to the bulk PRDF, even for the smallest diameter NP studied here, which is an indication of very small surface reconstruction and amorphization of the free surface of NPs (see Appendix A for more details).

\subsection{Vibrational density of states}

For the vibrational density of states (VDOS) of free or embedded NPs of different sizes, three methodologies are used here: 1 . the direct diagonalization of the dynamical matrix, 2. the eigenvalues estimation with the kernel polynomial method and 3 . the Fourier transform of the velocity autocorrelation function.

The first two methods are based on the dynamical matrix (DM). The elements of this matrix are defined for any periodical or finite object as:

$$
M_{i \alpha, j \beta}=\left(\sqrt{m_{j} m_{i}}\right)^{-1} \frac{\partial^{2} U}{\partial r_{i \alpha} \partial r_{j \beta}}
$$

with $i$ and $j$ indexing atoms, $\alpha$ and $\beta$ indexing the spatial directions, $m$ the masses of the atoms, $r_{j \beta}$ the position of the $j$ th atom displaced in the direction $\beta$, and $U$ the potential energy of the system. LAMMPS evaluates the DM by computing the force modification due to a single atomic displacement. The interactions between the atoms are reduced to their stiffness, as the energy landscape is harmonic. The DM associated with the 
full system (for FNPs) or only part of it (for ENPs) can be computed. This last one is computed by the motion of every atom belonging to a chosen set. The $\mathrm{SiO}_{2}$ atoms are fixed, which is equivalent to having a fixed interface around the embedded NPs, allowing the computation of VDOS only for the NPs. The DM describes the vibrations of the NPs with a single $3 N \times 3 N$ matrix with $N$ the number of atoms. The eigenvalues of this matrix give the eigenfrequencies square of the system and the eigenvectors allows us to obtain the vibrational modes. The handling of the matrix is facilitated by its sparseness: equation 1 reduces to 0 if the atom $i$ lies outside the cutoff radius of atom $j$.

For the smaller diameters NPs, one can diagonalize explicitly the dynamical matrix using the Implicitly Restarted Arnoldi Method [36] implemented in Sci Py for python. This method will be called "direct" diagonalization of the DM in the following. This method allows obtaining the vibrational modes and the frequencies simultaneously and thus one can compute the participation ratio $(\mathrm{PR})$ for each mode. The participation ratio indicates the fraction of atoms participating to a specific mode. It is computed as:

$$
P R=\frac{\left(\sum_{i=1}^{N}\left\|r_{i}\right\|^{2}\right)^{2}}{\sum_{i=1}^{N}\left\|r_{i}\right\|^{4}} \frac{1}{N}
$$

with $r_{i}$ the displacement of the $i$ th atom for the specific mode and $N$ the total number of atoms.

Another possibility to compute the VDOS is to use the Kernel Polynomial Method (KPM) described by Weie et al. [37] and adapted to the computation of VDOS by Beltukov et al. [38]. This method approximates the VDOS by series of Chebyshev polynomials.

The last among the three methods to obtain the VDOS is a statistical method involving the velocity autocorrelation function (VACF) [39]. The system is first thermalized at $50 \mathrm{~K}$ for $10 \mathrm{ps}$ with a Nos-Hoover thermostat. The temporal autocorrelation function of the velocities is then averaged over the particles and over the initial time step, using the same thermostat during the next $100 \mathrm{ps.}$. For these simulations, fixed boundary conditions are used for FNPs (that does not include any matrix around) and periodic boundary conditions are used for ENPs (at the borders of the surrounding matrix). The VDOS is then obtained from the temporal Fourier transform of the VACF. Finally, the Fourier transform is filtered with a Savitzky-Golay filter [40]. As the calculation of the VACF relies on statistics, it performs better for large systems over long-time simulation runs. On the other hand, the DM methods allow representing exactly every existing vibrational mode of the simplified (harmonic) system, but are limited to smaller systems due to restrictions related to the numerical diagonalization methods. The KPM method is a good alternative for large systems, but its precision depends on the number of iterations used. The VACF is thus simpler to handle, but less precise for small systems. Moreover, in case of ENPs, the VACF takes into account the atomic motions of the surrounding matrix, while this one is immobile in the DM methods. 
Vibrational density of states of free and embedded semiconducting GaN nanoparticles 6

\subsection{Thermal conductivity}

The thermal condutivity of the FNPs is computed thanks to the Equilibrium Molecular Dynamics method. This method is based on the fluctuation dissipation theorem, linking the decay of the fluctuation of an internal variable to its response function. The Green Kubo formula linking the flux auto-correlation integral to the thermal conductivity is written as:

$$
\kappa_{\alpha \beta}=\left(V k_{B} T^{2}\right)^{-1} \int_{0}^{\infty}\left\langle J_{\alpha}(0) J_{\beta}(t)\right\rangle d t
$$

with $\alpha$ and $\beta$ the directions, $V$ the volume of the NP, $k_{b}$ the Boltzman constant and $J_{\beta}(t)$ the heat flux in the direction $\beta$ at a time $t$. The heat flux computation entails for each atom a kinetic part and a potential part. The potential part is derived from the virial stress. A discretized version is [41]:

$$
\kappa_{\alpha \beta}=\Delta t\left(V k_{B} T^{2}\right)^{-1} \sum_{m=1}^{M}(m-p)^{-1} \sum_{n=1}^{p} J_{\alpha}(m+n) J_{\beta}(m)
$$

with $\Delta t$ the time step between two successive flux computations, $M$ the total number of time steps, and $p$ the maximum step over which the autocorrelation function is averaged. The inclusions are heated at $300 \mathrm{~K}$, using an initial velocity distribution without overall translational or rotational momentum. The system is then equilibrated at this temperature for $0.5 \mathrm{~ns}$ using a Nos-Hoover thermostat. We then extract the flux every $10 \mathrm{fs}$, and compute the flux autocorrelation decay over $40 \mathrm{ps}$, while the average is done over 5 to $10 \mathrm{~ns}$. To obtain better statistics, the results are averaged over 5 to 10 runs with different seeds for initial velocities. All these simulations were done at $300 \mathrm{~K}$ using a Nos-Hoover thermostat. The NPs were free to move, nevertheless, very few translation or rotation collective movements have been observed. These latter are excluded in the statistics for the computation of $\kappa$

There are known issues for the computation of virial stress with many-body potential such as Tersoff potential with LAMMPS, but as discussed by Termentzidis et al. [10] we will considered it as marginal.

\section{Results}

\subsection{VDOS of free nanoparticles}

The vibrational density of states of FNPs with different sizes (colored plain lines) and for bulk GaN (dashed black line) are depicted in figure 2 for comparison. At first view, one can notice that bulk and NPs VDOS are quite similar, even for small NPs composed of a few hundreds atoms (see table 1). The two acoustic peaks are noticeable and they can be linked to the Van Hove singularities [42]. Then, we will focus on the effect of the diameter reduction. Three main effects appear: the first one is the population of the bandgap, by 3 main modes at 14, 18 and $20 \mathrm{THz}$. Their intensities increase as the NPs decrease in size. Their relative importance in the DOS spectra is confirmed by the difference in participation ration $(\mathrm{PR})$ for different sizes: between $\mathrm{FNP}_{10}$ and $\mathrm{FNP}_{25}$ 
Vibrational density of states of free and embedded semiconducting GaN nanoparticles 7
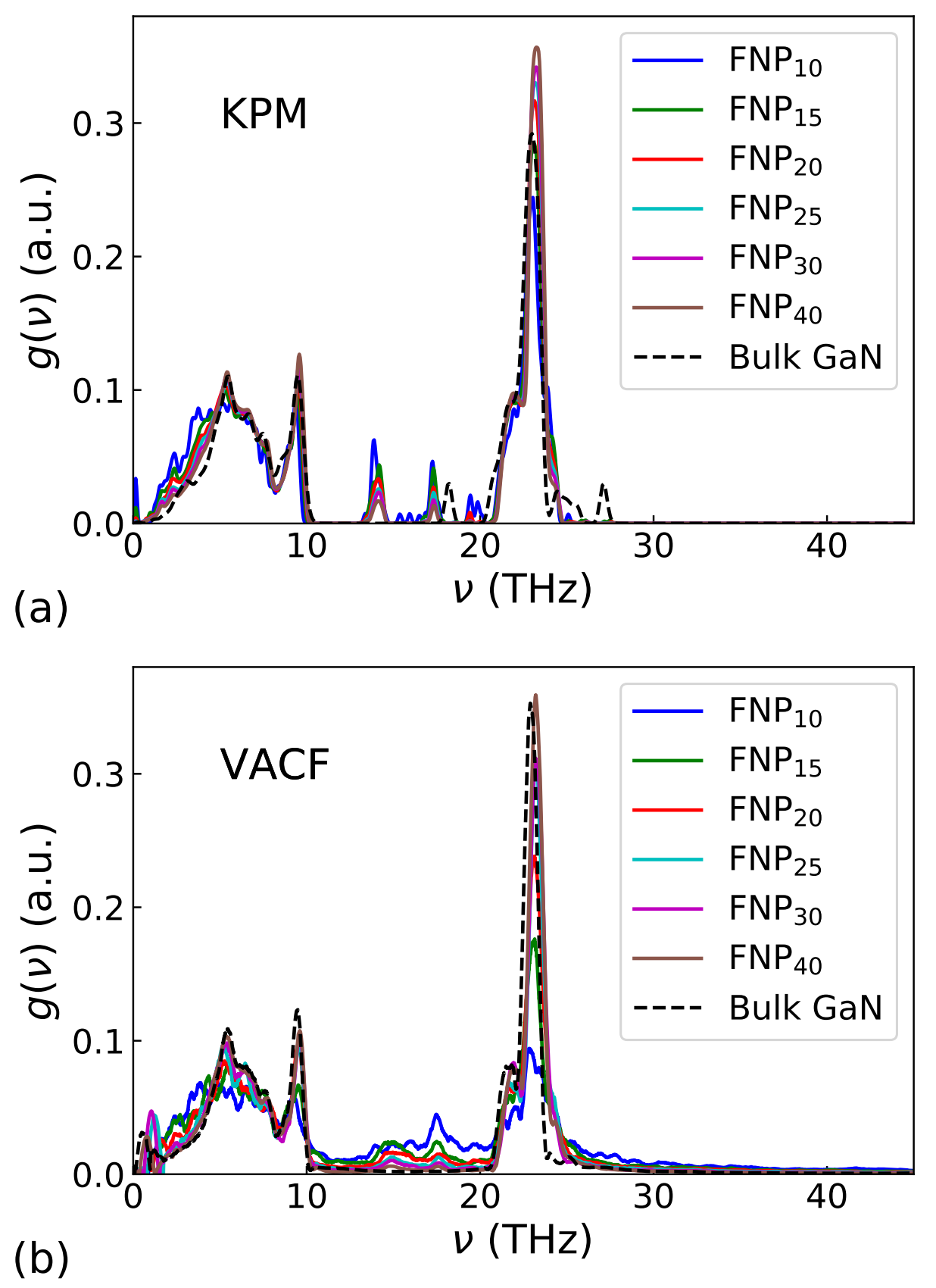

Figure 2: VDOS of GaN FNPs computed from the DM with KPM (a) and from the VACF (b). Colored lines represent the different NPs and the dashed black line the bulk $\mathrm{GaN}$

their PR decreases (see left column of figures 3), indicating that these modes are more and more spatially confined in percentage of in-phase moving atoms. These modes are related to surface modes and they were observed for other III-V semiconductors [22]. The increase of PR of those modes with the increase of the surface to volume ratio (see table 1) tends to confirm their localisation at the surface. It is also worth noticing here that the frequencies of these modes do not depend on the size of NPs. This means that 
Vibrational density of states of free and embedded semiconducting GaN nanoparticles 8

they cannot be linked to Lamb's modes or other resonant modes. Note also here, that with the VACF method (figure $2 \mathrm{~b}$ ) the peak at $14 \mathrm{THz}$ is less sharp.
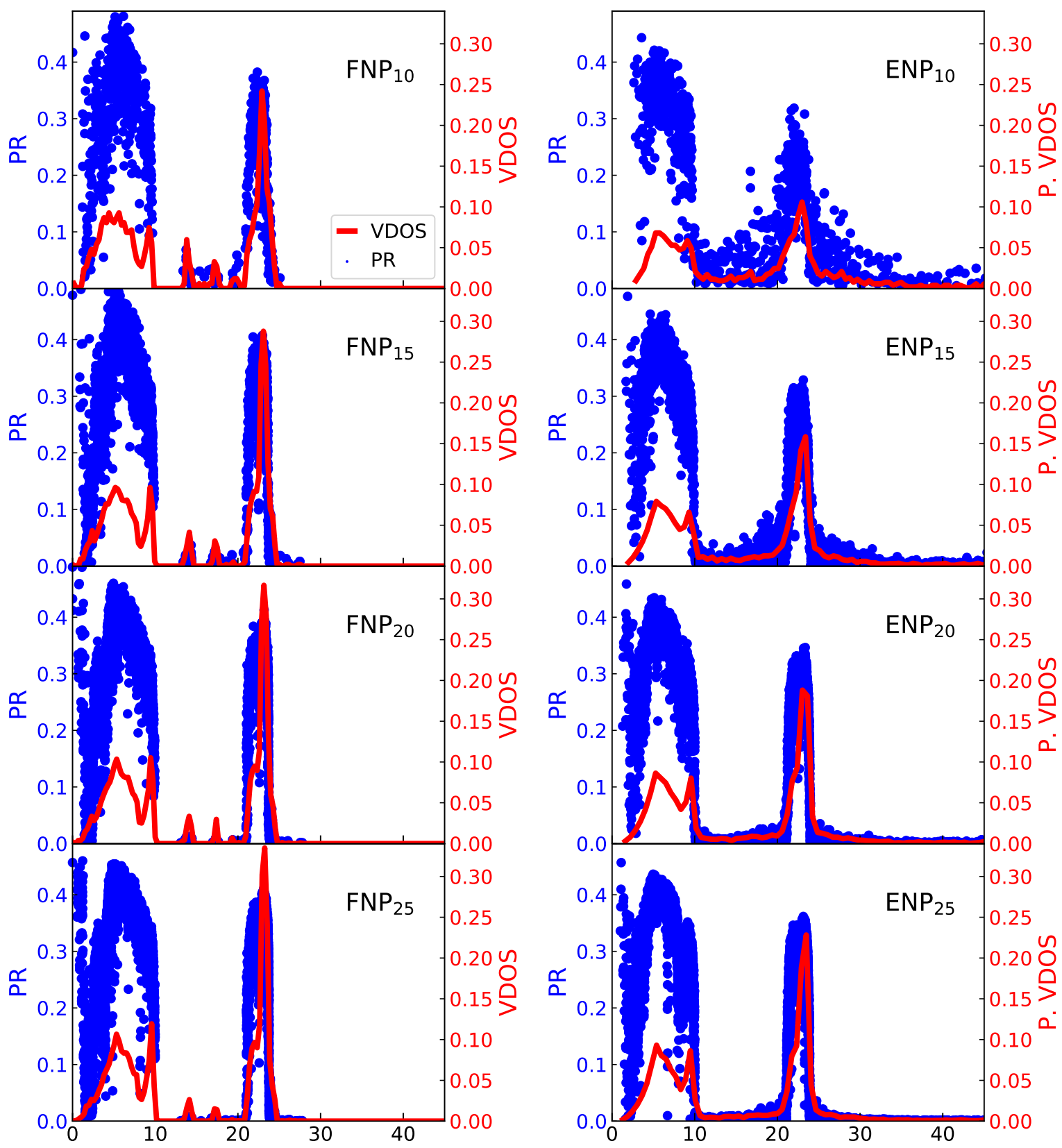

Figure 3: VDOS (red line) and participation ratio (blue dot) derived from the direct diagonalization of the DM for the FNPs (right) and ENPs (left)

The second effect is a redshift and/or blurring of the first acoustic peak for the radii up to $20 \AA$. This shift increases with decreasing NPs radius. This phenomenon is more sensitive to the VDOS estimation through KPM (figure 2a) and with the direct method (figure 3 left panel), but it is also visible when using the VACF, especially for the smallest NPs (figure 2b). This redshift differs from what was previously observed by Combe et al. [17] for Ge nanodots using Stillinger Weber potential. In the latter 
work a blue shift was mentioned instead, as it would be expected from the Lamb's theory where the eigenfrequencies scale with the inverse of the radius. Nevertheless, there are other studies that confirm our results; a mode softening. This softening was linked to the increased surface-to-volume ratio for metallic NP [21] or GaP NP [22]. Now concerning the blurring effect, we believe that this is due to the modification of the shape of resonant modes. If we use the longitudinal and transverse sound velocities given by Polian et al. [43], the fundamental Lamb's modes of a free isotropic sphere, corresponding to the NPs sizes of our study, lies between 1 and $5 \mathrm{THz}$. Below $3 \mathrm{THz}$ the wavelength of the longitudinal phonons as described by Jiang et al. [44] exceeds largely the smallest NP diameter. But, due to elastic heterogeneities, the effective sound velocity decreases, competing with the decrease in the NPs radius, and thus impacting the Lamb's frequencies [45].

The last diameter-dependent feature of VDOS is the intensity decrease of the optical peaks. A broadening of optical peaks was already observed and attributed to changes in the crystalline structure [22]. But the crystallinity of our NPs does not seem to change (see Appendix A). Moreover, the decrease of optical peaks intensities compared to acoustic ones appears also in the direct diagonalization. Thus, this phenomenon is independent of the method used to calculate the VDOS. The surface effect is the most probable explanation of the intensity decrease, as the ratio of Ga to $\mathrm{N}$ is constant for all the NPs.

Last but not least, it is worth to mention at this point, that the optic peaks obtained with this interatomic potential is blue shifted when compared to the one obtained from experimental results [46] and even more compared to ab-initio computation [47, 44]. The empirical potential used here shows a good agreement for the acoustic modes but is less accurate for the optic modes. A figure comparing the results from our study, the exprimental [46] and the ab-initio [47] results is available in Appendix B.

\subsection{Thermal conductivity of FNP}

The thermal conductivity $(\kappa)$ of the FNPs of radii 10, 15, 20, 25, 30 and $40 \AA$ are depicted in figure 4 and recorded in the table 1 . We have reported in the same table the percentage of surface atoms, belonging to a spherical shell of $2 \AA$ thickness. $2 \AA$ is chosen in order to encompass every atom appearing visually at the surface.

The $\kappa$ of NP, as expected, is only a small fraction of bulk GaN one $(\approx 160$ $220 \mathrm{~W} \mathrm{~K}^{-1} \mathrm{~m}^{-1}[48]$ ). For the smallest NP studied here, it is three orders of magnitude less than the bulk value. When increasing the size of NP, the $\kappa$ increases in a non linear way, in contrast to a previous study for Silicon FNPs with diameters 2 to $12 \mathrm{~nm}$ in which a linear size dependence was reported [8].

The previously reported VDOS modifications due to the size effect can impact the $\kappa$ when taking into account the separate contribution of each phonon frequency. For instance, modes below $5 \mathrm{THz}$ contribute to $50 \%$ of the thermal conductivity according to Tang et al. [47]. Furthermore, the surface modes in the bandgap gain intensity upon 
Vibrational density of states of free and embedded semiconducting GaN nanoparticles10

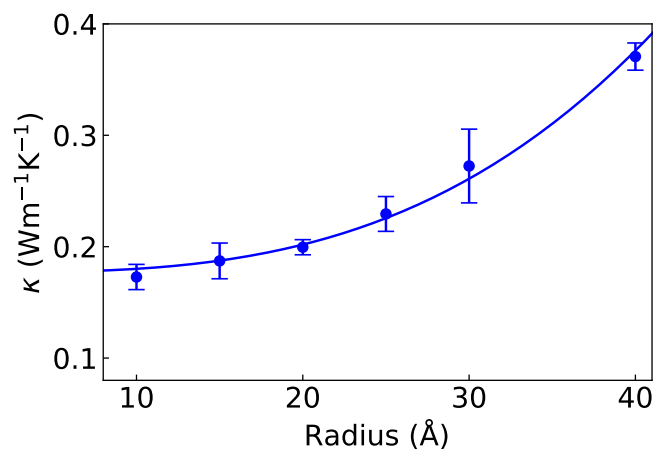

Figure 4: Thermal conductivity of FNPs computed with EMD at 300K, the error bar corresponds to the standard deviation between the 5 to 10 independent runs. The blue line is a fit to a cubic scaling law as a guide to the eye.

\begin{tabular}{|c|c|c|c|}
\hline & \# of atoms & \% of surface atom & $\kappa\left(\mathrm{W} \mathrm{K}^{-1} \mathrm{~m}^{-1}\right)$ \\
\hline$N P_{10}$ & 369 & 52 & $0.17 \pm .01$ \\
\hline$N P_{15}$ & 1239 & 36 & $0.19 \pm .02$ \\
\hline$N P_{20}$ & 2952 & 29 & $0.20 \pm .01$ \\
\hline$N P_{25}$ & 5657 & 24 & $0.23 \pm .02$ \\
\hline$N P_{30}$ & 9942 & 20 & $0.27 \pm .03$ \\
\hline$N P_{40}$ & 23580 & 16 & $0.37 \pm .02$ \\
\hline
\end{tabular}

Table 1: Characteristics of the FNP

decreasing the radius of NPs. However, these surface modes do not penetrate in the NPs, so they do not contribute much to the overall flux. Size effects are also known to promote phonon mode hybridization that can impact the thermal conductivity [49]. We are aware that the few points in figure 4 cannot give the exact tendency nor the whole picture, but we can notice a cubic scaling dependence of $\kappa$ with the diameter, which might be related to a volume $\left(r^{3}\right)$ dependence. The calculated thermal conductivity of a FNP here quantifies how effective the energy exchange inside the particle is. Due to size effects, it differs from the bulk. This is an important effect that one should take into account when effective medium approximation (EMA) models are used to estimate the effective $\kappa$ of a nanocomposite.

\subsection{Vibrational density of state of ENP}

In the following section the ENP will be studied, focusing on the substitution of the free surface by an interface with an amorphous material. For the case of ENPs, the frequency shift of the VDOS acoustic peaks with the size of the ENPs as observed in the previous paragraph for the FNPs disappears, as well as the modes inside the bandgap of bulk GaN at 14, 18 and $20 \mathrm{THz}$. We relate these modifications to the elimination of the surface modes [21, 22]. 
Vibrational density of states of free and embedded semiconducting GaN nanoparticles11
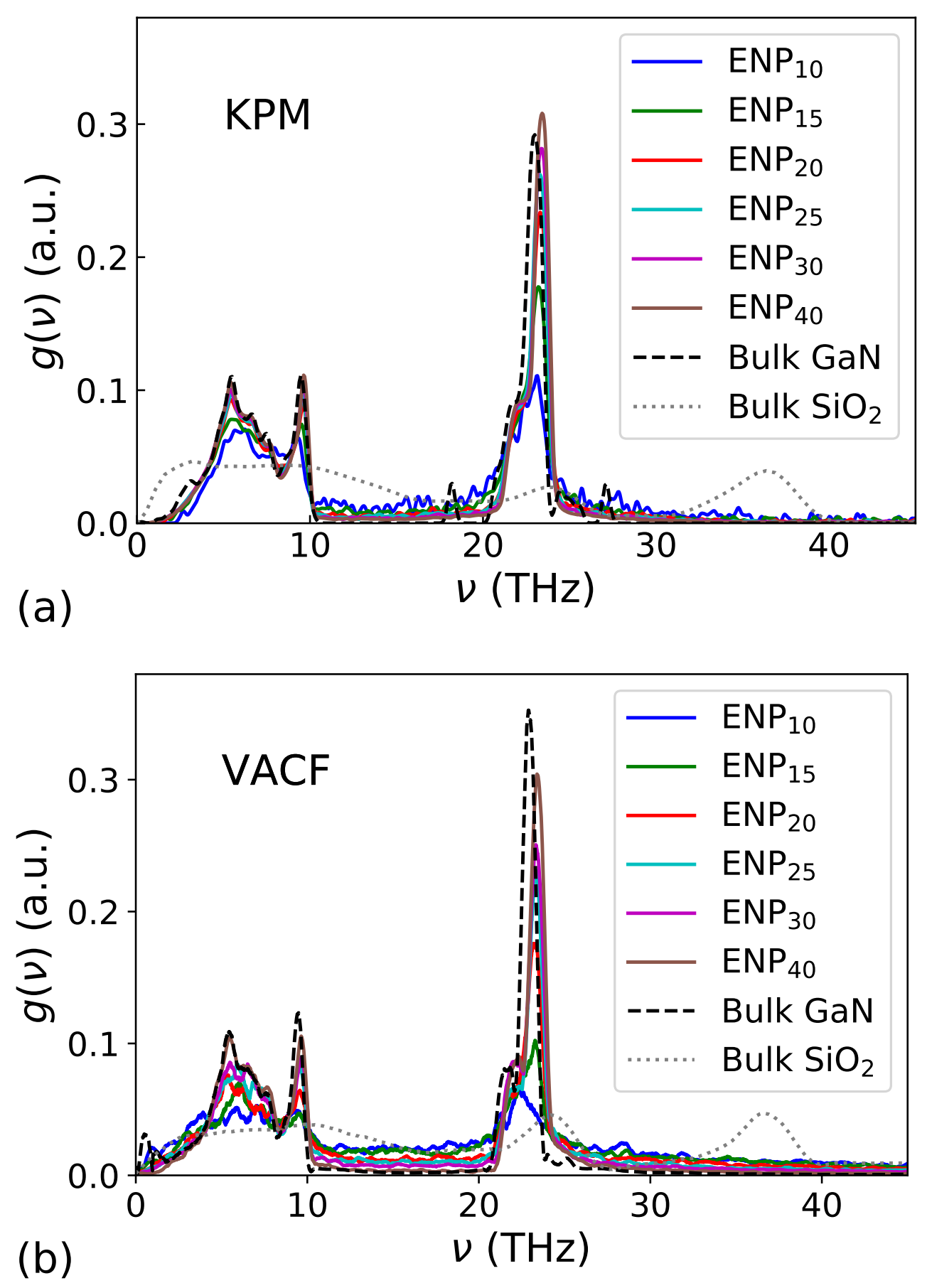

Figure 5: VDOS of GaN ENPs computed with the DM with KPM (a) and from the VACF (b). Colored lines represent the different NPs, the dashed black line the GaN bulk and the dotted gray line the $\mathrm{SiO}_{2}$.

A marked broadening of the optical peaks can still be observed for the smallest ENPs, as well as a population of the bandgap, but this time in a much more uniform way. This can be understood as the influence of the $\mathrm{SiO}_{2}$ matrix on the modes. Matrix modes are indeed available at those frequencies as can be seen in figure 5 where the $\mathrm{SiO}_{2}$ 
Vibrational density of states of free and embedded semiconducting GaN nanoparticles12

VDOS is represented with a dotted line. Previous work showed that, at the interfaces, modes of the different phases combine together $[50,51,52]$. This effect could be used to deduce which mode can be transmitted or reflected on the inclusion. Additionally, new optical branches seem to appear towards high frequencies for all ENP sizes, something that was not observed for FNP. The most probable explanation for this might be the hybridization of the optical modes of the GaN with the higher frequencies modes of the $\mathrm{SiO}_{2}$ matrix.

Finally, the only common tendency between free and embedded NPs is the relative decrease of the optical peaks for small sizes. This effect is even more visible in figure 5 compared to figure 2. It is partially due to the normalization of the VDOS area, but the acoustic peaks seem less impacted. As this effect is present for both free and embedded NPs, it cannot be due to free surface effect only. It is most probably related to the amorphization of the surface or interface. Indeed, the embedded NPs seem to show more amorphization than the FNPs, as discussed in Appendix A.

The previous observation on the VDOS of ENPs are valid for the two methodologies VACF and KPM. The last one shows a sharper definition of modes and it is thus selected to make a more rigorous comparison between the ENPs and FNPs VDOS. The comparison between FNPs and ENPs in figure 6 allows underlining the previously cited differences (here represented in red). First the difference in intensity of the optical peaks between the FNPs and ENPs can be observed; the peaks for the ENPs are lower compared to FNPs. This difference is enhanced when decreasing the size of the NPs (red arrows). Then the redshift of the TA peak for FNPs appears clearly for radii up to $20 \AA$, while the distinct modes in the bandgap for FNPs disappear for the ENPs. This confirms the fact, mentioned in the previous paragraph, that these modes are related to free surface effects. Finally, one can observe in ENPs background modes, which are related to the matrix.

\section{Discussion and Conclusion}

Let's start this section with an overall comparison of the 3 methods used to simulate the VDOS for both embedded and free NPs. To do so, the VDOS of NPs with two different sizes are depicted in figure 7 for the 3 methods. The estimations of the DOS with the direct diagonalization or the approximation with KPM leed to sharper features in the DOS, especially for the smallest diameters NPs (top panels of figure 7). This can have two origins: the poor statistics of the VACF due to the reduced number of particles, and/or the anharmonicity mainly due to the agitation of surface atoms during simulations. In case of ENPs, the vibrations of the surrounding matrix allowed only in the VACF method, contribute also to broaden the VDOS in this case. For bigger diameters, the similarities between the methods using the DM and the VACF validate the fixed interface conditions used when computing the DM. Finally, this shows that the computation of the DM restricted to the NP atoms only is globally adapted to the computation of VDOS of NPs embedded in an amorphous matrix. 
Vibrational density of states of free and embedded semiconducting GaN nanoparticles13

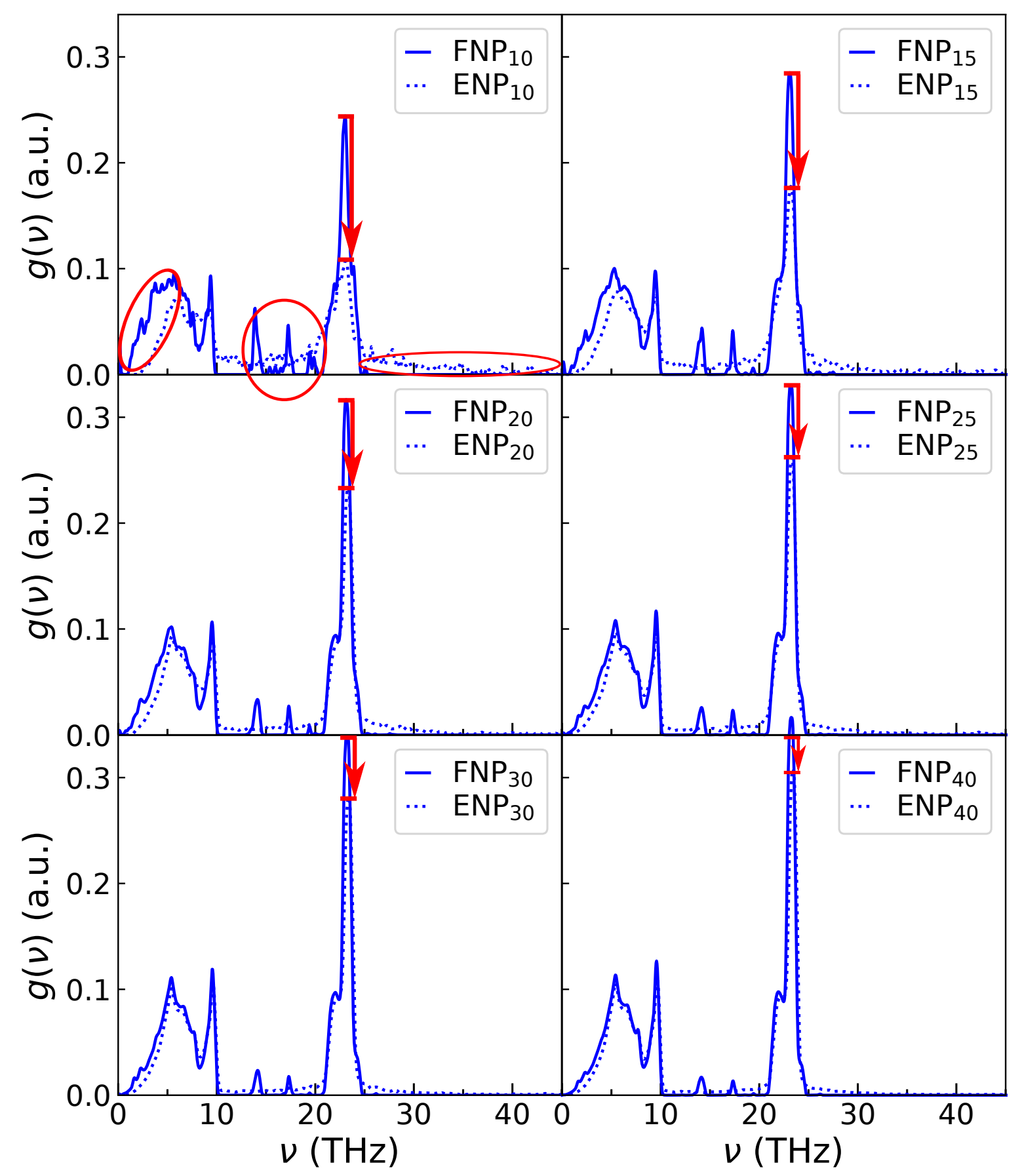

Figure 6: VDOS comparison for every NP diameter between free in continuous line and embedded in dotted line, from the DM using KPM

In general the three methods converge for NPs with diameters larger than $5 \mathrm{~nm}$. Nevertheless, for smaller NPs one should be careful about the methodology selection, as the impact of temperature can only be taken into account directly with the VACF method. On the other hand the DM approach detects detailed features that the VACF looses due to thermal agitation and poor statistics (small number of atoms). We have 
Vibrational density of states of free and embedded semiconducting GaN nanoparticles14

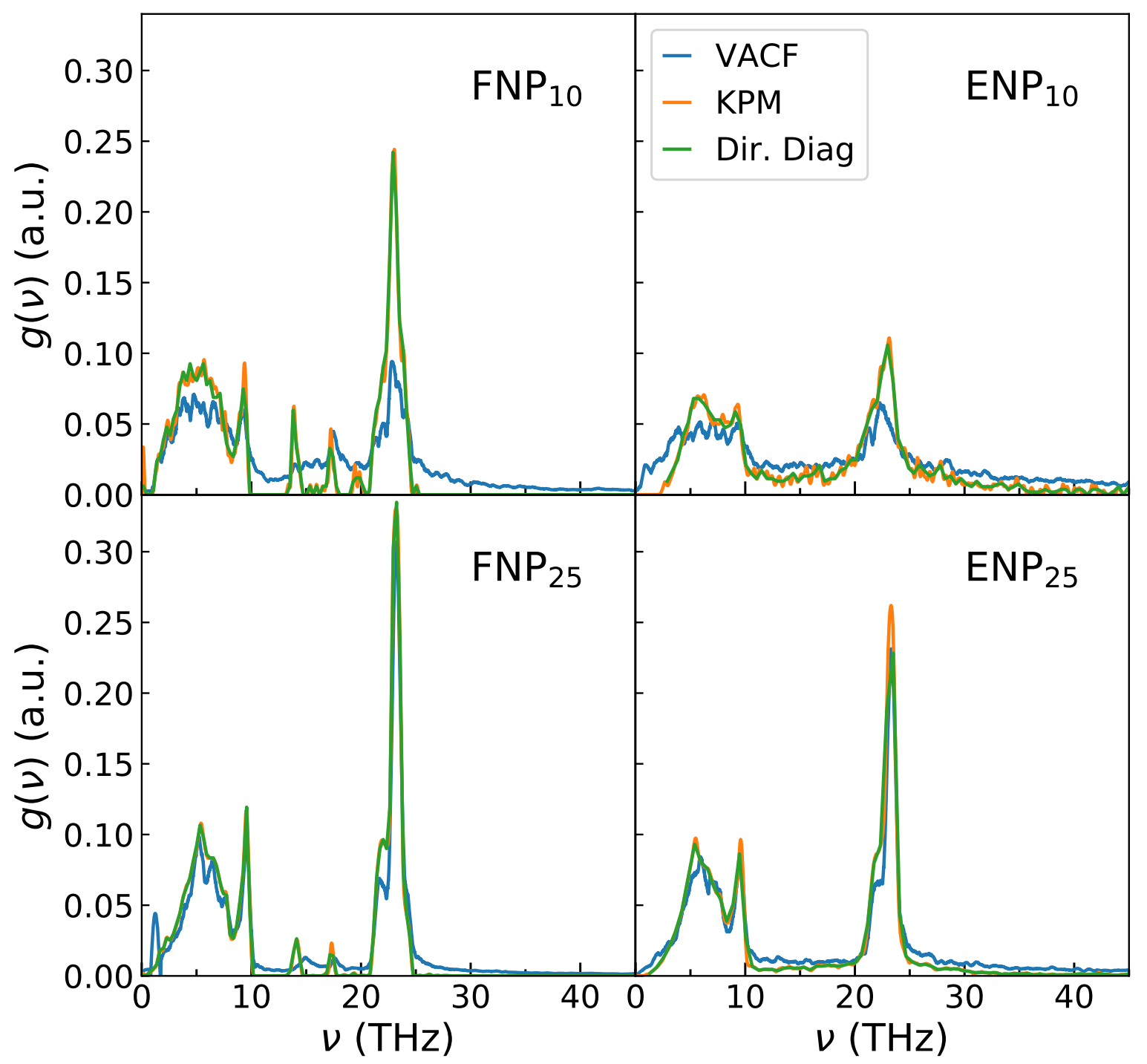

Figure 7: VDOS for $\mathrm{NP}_{10}$ and $\mathrm{NP}_{25}$ free and embedded, using the different method for the evaluation of the VDOS

to notice here that the DM is feasible only for small NPs with maximum 10000 atoms because larger number cannot be considered due to CPU limitations related to the direct diagonalization scheme. In contrast KPM, scheme can overpass the direct method limitations, but it is also constrained due to matrix size limitation compared to available RAM.

To conclude, the vibrational density of states of GaN NPs free or embedded in an $\mathrm{SiO}_{2}$ matrix was studied. We have observed 3 different phenomena for the FNP: a redshift of the first acoustic peak, the appearance of surface modes in the bandgap and an intensity decrease of the optical modes. As the NP is embedded in a $\mathrm{SiO}_{2}$ matrix, only the latter effect persists, and modes induced by the interface with the matrix increase in intensity as the NPs decrease in size. We did not observe specific peaks for 


\section{Vibrational density of states of free and embedded semiconducting GaN nanoparticles15}

the Lambs modes characterized by a frequency inversely proportional to the radius of the inclusions: they are indeed hidden in the VDOS spectra, but can be identified easily at low frequencies with the DM.

Concerning the thermal conductivity of the FNPs, we have observed that it does not follow a previous linear dependence observed for the case of silicon NPs. In III$\mathrm{V}$ semiconducting NPs and more specifically in GaN NPs the thermal conductivity increases in a cubic power low of nanoparticles diameter. The nonlinear size dependence of the thermal conductivity underlines that the mean free path limitation to the NPs size is not the only parameter to take into account, otherwise we would have had a linear dependence with the size. This nonlinearity might be due to surface modes and how these modes interact with the heat flux fluctuations in the core of the NP. Note that collective modes involving the full matrix coupled to the nanoparticle are not taken into account by the DM methods due to the fixed condition. It was shown in recent articles that the matrix can also play a role [51], especially from its damping properties [11], together with the impedance break between the particle and the matrix [52]. Collective modes may appear when nanoparticles are embedded in a host matrix. However, our purpose here focuses on the properties of isolated nanoparticles. The thermal properties of the specific nanocomposite ( $\mathrm{GaN}$ nanoinclusions in a silica matrix) have been already studied and discussed elsewhere [10].

\section{Acknowledgments}

This work was granted access to the HPC resources of IDRIS under the allocation 2020A0070911092 made by GENCI. We would like to thank Professor Joseph Kioseoglou of the Aristotle Universtity of Thessaloniki for all fruitful discussions.

\section{Appendix A. Partial radial distribution function}
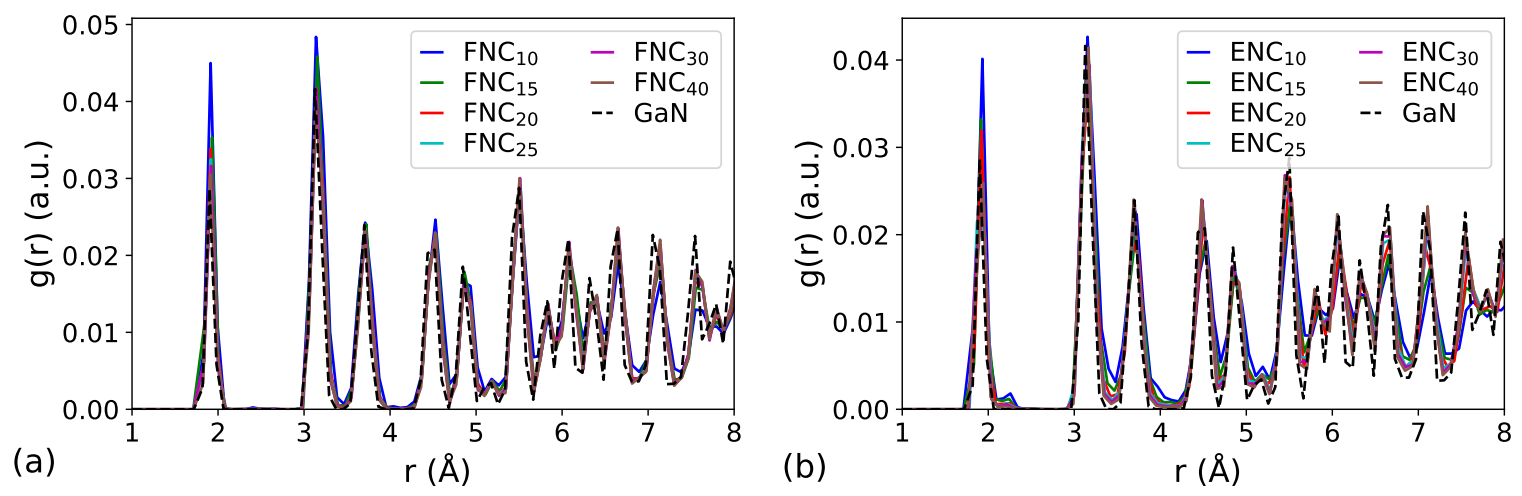

Figure A1: RDF for GaN atoms at 300K for FNP (a) and partial RDF of GaN in ENP (b)

The PRDF of ENPs and FNPs is represented in figure A1. The PRDF allows 


\section{Vibrational density of states of free and embedded semiconducting GaN nanoparticles16}

comparing the crystalline structure of the NPs with decreasing radius and the differences with the bulk. The first peak represents the distance to the nearest neighbor, the second one the distance to the second nearest neighbor and so one. Here, we see little or no change to the PRDF going from bulk to free NPs. A similar analysis made on Si NPs with a Tersoff potential showed reorganization at the surface [7], this discrepancy may come from the higher temperature $(1600 \mathrm{~K})$ annealing of their NPs. For the embedded NPs on the opposite, a peak broadening can be observed. It is visible from the second nearest neighbor on. This broadening increases with decreasing diameter which is symptomatic of amorphization at the interface due to the contact with a-SiO $\mathrm{S}_{2}$. These observations confirm that for our configuration, the $\mathrm{GaN}_{-} \mathrm{SiO}_{2}$ interface has more impact on the vibrational properties than the free surface of FNPs.

\section{Appendix B. Comparison of GaN bulk VDOS}

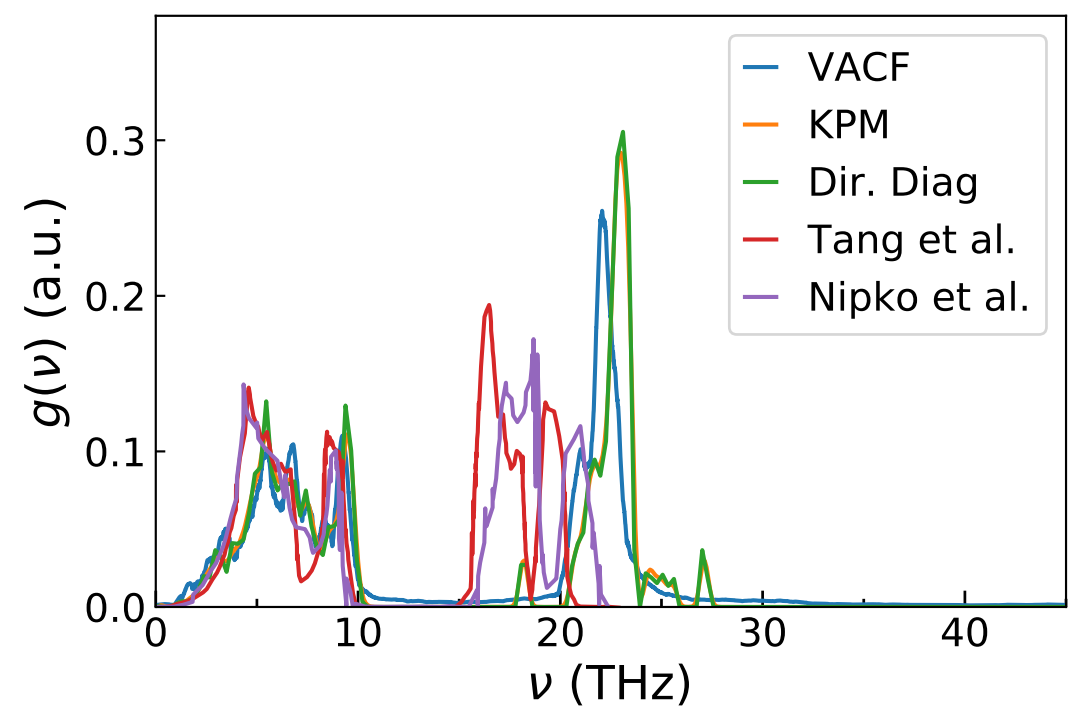

Figure B1: Comparison of the VDOS of bulk GaN using KPM, direct diagonalization and VACF with results from ab-initio calculation by Tang et al. [47] and experimental results by Nipko et al. [46]

\section{References}

[1] Qiu L, Zhu N, Feng Y, Michaelides E E, Żyła G, Jing D, Zhang X, Norris P M, Markides C N and Mahian O 2019 Physics Reports ISSN 03701573 URL https://doi.org/10.1016/j.physrep.2019.12.001

[2] Daudin B 2008 Journal of Physics Condensed Matter 20 ISSN 09538984

[3] Nabati Shoghl S, Jamali J and Keshavarz Moraveji M 2016 Experimental Thermal and Fluid Science $\mathbf{7 4} \quad 339-346 \quad$ ISSN $08941777 \quad$ URL http://dx.doi.org/10.1016/j.expthermflusci.2016.01.004

[4] Del Castillo-Castro T, Larios-Rodriguez E, Molina-Arenas Z, Castillo-Ortega M M and Tanori J 2007 Composites Part A: Applied Science and Manufacturing 38 107-113 ISSN 1359835X 
[5] Berweger S, Atkin J M, Xu X G, Olmon R L and Raschke M B 2011 Nano Letters 11 4309-4313 ISSN 15306984

[6] Girard A, Gehan H, Crut A, Mermet A, Saviot L and Margueritat J 2016 Nano Letters $163843-$ 3849 ISSN 15306992

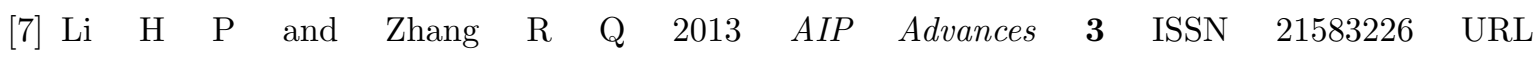
https://doi.org/10.1063/1.4818591

[8] Fang K C, Weng C I and Ju S P 2006 Nanotechnology 17 3909-3914 ISSN 09574484

[9] Nomura M, Shiomi J, Shiga T and Anufriev R 2018 Japanese Journal of Applied Physics 57 ISSN 13474065

[10] Termentzidis K, Giordano V M, Katsikini M, Paloura E, Pernot G, Verdier M, Lacroix D, Karakostas I and Kioseogloud J 2018 Nanoscale 10 21732-21741 ISSN 18196608

[11] Tlili A, Giordano V M, Beltukov Y M, Desmarchelier P, Merabia S and Tanguy A 2019 Nanoscale 11 21502-21512 URL http://dx.doi.org/10.1039/C9NR03952J

[12] Huang C L, Qian X and Yang R G 2017 Epl 117 ISSN 12864854

[13] Eringen A C and Suhubi E 1975 Elastodynamics academic ed vol II (New York) ISBN 0122406028

[14] Juvé V, Crut A, Maioli P, Pellarin M, Broyer M, Del Fatti N and Vallée F 2010 Nano Letters 10 1853-1858 ISSN 15306984

[15] Wittmer J P, Tanguy A, Leonforte F and Barrat J L 2002 Europhysics Letters 57 423-429 ISSN 01694332

[16] Saviot L and Murray D B 2009 Physical Review B - Condensed Matter and Materials Physics 79 1-11 ISSN 10980121 (Preprint 0904.2959)

[17] Combe N, Huntzinger J R and Mlayah A 2007 Physical Review B - Condensed Matter and Materials Physics 76 1-12 ISSN 10980121

[18] Murray D B and Saviot L 2004 Physical Review B - Condensed Matter and Materials Physics 69 1-9 ISSN 1550235X

[19] Lamb H 1881 Proceedings of the London Mathematical Society 1 189-212

[20] Sauceda H E, Salazar F, Pérez L A and Garzón I L 2013 Journal of Physical Chemistry C 117 25160-25168 ISSN 19327447

[21] Calvo S R and Balbuena P B 2005 Surface Science 581 213-224 ISSN 00396028

[22] Han P and Bester G 2011 Physical Review B - Condensed Matter and Materials Physics 83 1-8 ISSN 10980121

[23] Han P and Bester G 2012 Physical Review B - Condensed Matter and Materials Physics 85 1-4 ISSN 10980121 (Preprint 1202.1634)

[24] Kurban M, Baris Malcioglu O and Erkoç S 2016 Chemical Physics 464 40-45 ISSN 03010104

[25] Kurban M 2018 Turkish Journal of Physics 42 443-454 ISSN 13036122

[26] Plimpton S 1995 Journal of Computational Physics 117 1-19 ISSN 00219991 (Preprint nag. 2347)

[27] Kioseoglou J, Katsikini M, Termentzidis K, Karakostas I and Paloura E C 2017 Journal of Applied Physics 121 ISSN 10897550

[28] Munetoh S, Motooka T, Moriguchi K and Shintani A 2007 Computational Materials Science 39 334-339 ISSN 09270256

[29] Nord J, Albe K, Erhart P and Nordlund K 2003 Journal of Physics Condensed Matter 155649 5662 ISSN 09538984

[30] Okeke O U and Lowther J E 2009 Molecular dynamics of binary metal nitrides and ternary oxynitrides URL http://dx.doi.org/10.1016/j.physb.2009.06.003

[31] de Brito Mota F, Justo J and Fazzio A 1998 Physical Review B - Condensed Matter and Materials Physics 58 8323-8328 ISSN 1550235X

[32] Stukowski A 2010 Modelling and Simulation in Materials Science and Engineering 18015012

[33] Mantisi B, Tanguy A, Kermouche G, Barthel E, Mantisi B, Tanguy A, Kermouche G and Barthel E 2012 Eur. Phys. J. B (2012) 85304

[34] Van Beest B W H, Kramer G J, Shell K and Van Santen R A 1990 1955-1958 URL https://journals.aps.org/prl/pdf/10.1103/PhysRevLett.64.1955 
[35] Liang Y, Miranda C R and Scandolo S 2007 Physical Review B 1-5

[36] Lehoucq R B, Sorensen D C and Yang C 1998 ARPACK users' guide: solution of large-scale eigenvalue problems with implicitly restarted Arnoldi methods vol 6 (Siam)

[37] Weiße A, Wellein G, Alvermann A and Fehske H 2006 Reviews of Modern Physics 78 275-306 ISSN 15390756

[38] Beltukov Y M, Fusco C, Parshin D A and Tanguy A 2016 Physical Review E 93 1-18 ISSN 24700053 (Preprint 1510.01761)

[39] Dove M 1993 Introduction to lattice dynamics vol 136

[40] Savitzky A and Golay M J 1964 Analytical chemistry 36 1627-1639

[41] Schelling P K, Phillpot S R and Keblinski P 2002 Nanoscale thermal transport Journal of Applied Physics 802484 URL http://aip.scitation.org/toc/apl/80/14

[42] Van Hove L 1953 Physical Review 891189

[43] Polian A, Grimsditch M and Grzegory I 1996 Journal of Applied Physics 79 3343-3344 ISSN 00218979

[44] Jiang Y, Cai S, Tao Y, Wei Z, Bi K and Chen Y 2017 Computational Materials Science 138 419-425 ISSN 09270256 URL http://dx.doi.org/10.1016/j.commatsci.2017.07.012

[45] Tanguy A, Wittmer J P, Leonforte F and Barrat J 2002 Physical Review B - Condensed Matter and Materials Physics 1-17

[46] Nipko J C, Loong C K, Balkas C M and Davis R F 1998 Applied Physics Letters 73 34-36 ISSN 00036951

[47] Tang D S, Qin G Z, Hu M and Cao B Y 2020 Journal of Applied Physics 127 ISSN 10897550

[48] Jezowski A, Danilchenko B A, Boćkowski M, Grzegory I, Krukowski S, Suski T and Paszkiewicz T 2003 Solid State Communications 128 69-73 ISSN 00381098

[49] Ma, Anufriev, RomanNomura M, Shiomi J and Shiga T 2018 Japanese Journal of Applied Physics 57 ISSN 13474065

[50] France-Lanord A, Merabia S, Albaret T, Lacroix D and Termentzidis K 2014 Journal of Physics Condensed Matter 26 ISSN 1361648X

[51] Damart T, Giordano V M and Tanguy A 2015 Physical Review B 92 1-11 ISSN 1550235X (Preprint 1507.02112)

[52] Luo H, Gravouil A, Giordano V and Tanguy A 2019 Nanomaterials 9 ISSN 20794991 